\title{
Efecto del calor aportado en recargues nanoestructurados base hierro
}

\section{(Effect of Heat Input on the Fe-based Nanostructured Weld Overlay)}

\author{
Agustín Gualco ${ }^{1}$, Hernán G. Svoboda ${ }^{2,3}$, Estela S. Surian ${ }^{1,4}$ \\ ${ }^{1}$ Universidad Nacional de Lomas de Zamora, Facultad de Ingeniería, Secretaría de Investigación, Buenos Aires, Argentina. e-mail: \\ agustingualco@yahoo.com.ar \\ ${ }^{2}$ Universidad de Buenos Aires, Facultad de Ingeniería, Laboratorio de Materiales y Estructuras y Departamento de Ingeniería \\ Mecánica, Laboratorio de Materiales y Estructuras, INTECIN, Argentina. \\ ${ }^{3}$ Consejo Nacional de Investigaciones Cientificas y Técnicas, Argentina. \\ ${ }^{4}$ Universidad Tecnológica Nacional, Facultad Regional San Nicolás, DEYTEMA-Centro de Desarrollo y Tecnología de Materiales, \\ San Nicolás, Argentina.
}

\begin{abstract}
Resumen
En los últimos años se han desarrollado consumibles de soldadura que depositan recubrimientos duros de aleaciones base hierro nanoestructuradas de gran resistencia al desgaste abrasivo. Las resistencias al desgaste erosivo y abrasivo están controladas principalmente por la composición química y la microestructura. A su vez, la microestructura del metal depositado puede presentar variaciones con el procedimiento de soldadura empleado, especialmente en relación al aporte térmico. Los parámetros operativos que definen el aporte térmico (tensión, corriente y velocidad de soldadura) afectan aspectos como la geometría del cordón (ancho, penetración y sobremonta) y la dilución con el material base. El propósito de este trabajo fue estudiar el efecto del calor aportado sobre las características geométricas del cordón, la dilución y la evolución microestructural de una aleación nanoestructurada base hierro, depositada por FCAW. Se soldaron muestras con aportes térmicos de entre 0,5 y 3,5 kJ/mm. Sobre cada cupón soldado se realizó un relevamiento dimensional, se analizó la composición química y se caracterizó la microestructura usando microscopías óptica y electrónica de barrido y difracción de rayos X. También se midieron la microdureza del depósito, el tamaño de cristalita y el grado de dilución. Se observó una gran influencia de las condiciones de proceso sobre la geometría del cordón. La dilución varió entre un 30 y un 40\%, la microdureza del depósito se encontró entre 800 y $870 \mathrm{HV} 1$ y el tamaño de cristalita osciló entre 105 y 130 nm, en función de las variables de proceso empleadas. Las mayores durezas y los menores tamaños de cristalita se obtuvieron con el menor aporte térmico, asociado a una menor dilución.
\end{abstract}

Palabras claves: recubrimientos duros, nanomateriales, calor aportado, microestructura.

\begin{abstract}
In the last years there have been developed several consumables that deposit hard nanostructured iron-based metals with high resistance to abrasive wear. The erosive and abrasive wear resistances are mainly controlled by the chemical composition and the microstructure. In turn, the microstructure of the deposited metal usually shows variations with the experimental welding procedure, particularly related to the heat input. Also, the operating parameters that define the heat input (voltage, current and welding speed) affect aspects such as bead geometry (wide, penetration and reinforcement) and dilution with the base metal of the bead. The purpose of this work was to study the effect of heat input on the geometry of the bead, the dilution and the microstructural characteristics of a nanostructured iron-based alloy deposited by FCAW. Several samples with heat input between 0.5 and $3.5 \mathrm{~kJ} / \mathrm{mm}$ were welded. Chemical composition was determined, microstructure was studied using both optical and electronic microscopy and X ray diffraction; hardness, grain size and percentage of dilution were measured. The microhardness of the deposit was found between 800 and 870 HV1, grain size variations were between 105 and $130 \mathrm{~nm}$ and the dilution ranged between 30 and 40\%, depending on process variables used. The highest hardness and the lowest cristallite sizes were obtained with the lowest heat input, associated to a lower dilution.
\end{abstract}

Key-words: hardfacing; nanomaterials, heat input, microstructure.

Recebido em 11/07/2013, texto final em 18/09/2013.

\section{Introducción}

La tecnología de los materiales para recubrimientos superficiales ha experimentado un significativo progreso en los últimos años, desarrollándose continuamente revestimientos 
específicos para demandas particulares. En este aspecto, el estudio sistemático de los consumibles y los procedimientos de soldadura para aplicación de revestimientos duros, es de gran interés para la optimización del diseño de dichos consumibles y para la evaluación y puesta a punto de los mencionados procedimientos de soldadura. Dentro de este contexto, el calor aportado, la composición del gas de protección, la temperatura de pre-calentamiento y entre pasadas y el tratamiento térmico post-soldadura son algunas de las variables más relevantes del procedimiento de soldadura $[1,2]$.

Los depósitos en estudio son de muy alta dureza, con precipitados de borocarburos, carburos y boruros complejos ultraduros distribuidos en una matriz $\alpha$-ferrítica nanoestructurada. Durante el enfriamiento del metal fundido, a pesar de que el movimiento al azar de los átomos genera grupos de ellos dispersos y no ordenados, durante la solidificación, la mayoría se acomoda en una estructura regular predeterminada, cristalina. Excepcionalmente, bajo enfriamiento muy rápido ciertas aleaciones pueden "congelarse" reteniendo su arreglo líquido atómico, debido al insuficiente tiempo para su "ordenamiento", obteniéndose así una estructura vítrea superenfriada con inusuales propiedades. Esta "Rapid Solidification Technology" se utiliza para la generación de capas metálicas a escala microscópica que combinan alta resistencia, módulo elástico y resistencia a la corrosión. Sin embargo esta tecnología no es adecuada para producir estructuras amorfas a escala macroscópica en las que el espesor útil de recargue no se requiere en micrones sino en milímetros. Para este último caso se descubrió que si varios y diferentes elementos con radios atómicos muy distintos se funden juntos, al solidificar, la aleación resultante tiene grandes dificultades para nuclear y crecer según el ordenamiento cristalino normal y lo que se obtiene son granos de tamaño nanométrico en una microestructura "Nano Alloy" [3], un estado de agregado atómico entre el ordenado cristalino y el completamente desordenado, característico del estado líquido. Los materiales así obtenidos aplicables a depósitos por soldadura presentan excepcional resistencia a la abrasión y la erosión, depósitos de una sola capa con durezas extremadamente altas ( $70 \mathrm{HRc}$ ), bajo coeficiente de fricción sin lubricación y cordones de superficie suave.

A partir de recientes avances en el área de soldadura se han desarrollado alambres tubulares que permiten depositar aleaciones de hierro con una matriz de $\alpha$-ferrita nanoestructurada con carburos y boruros precipitados [2,3]. Estos materiales poseen una elevada dureza asociada al extremadamente pequeño tamaño de cristalita de la matriz, del orden de 30 a $50 \mathrm{~nm}$. Cabe aclarar, que cristalita es un sólido compuesto con átomos dispuestos en un patrón periódico en tres dimensiones cuya medición se realiza con difracción de rayos x. Si dicha medición se hubiera realizado por microscopia electrónica de transmisión se denomina grano [4]. Además, estos materiales presentan en general precipitados ultra duros, como carburos de niobio, boro (o carboboburos de cromo) o tungsteno que mejoran la resistencia al desgate abrasivo. Se aplican sobre superficies nuevas o sobre piezas desgastas de equipos, proporcionando propiedades específicas como pueden ser resistencias al desgaste abrasivo y adhesivo, a la erosión, a la corrosión, al calor y sus combinaciones [1]. En base a recientes estadísticas se estima que el $50-60 \%$ de los elementos de equipos se desgastan debido a erosión y/o abrasión (de baja presión, alta presión, en seco o húmedo) $[1,2,5]$ por lo que su aplicación presenta un fuerte impacto en la vida de componentes.

La resistencia al desgaste abrasivo de depósitos obtenidos por FCAW depende de muchos factores, pero principalmente son la composición química y la microestructura los que definen sus propiedades [2]. A su vez, el calor aportado, que depende de la velocidad de soldadura, la corriente y la tensión de arco y que define el ciclo térmico, influye fuertemente sobre la estructura de solidificación del material depositado y además controla la forma de la línea de fusión, la profundidad de penetración y la dilución del depósito. Trabajos previos [6-8] han mostrado que el aumento del calor aportado produce cambios en la composición química del metal depositado, en su microestructura y un aumento del efecto térmico entrepasadas y por ende variaciones en las propiedades mecánicas finales del depósito. En depósitos de aleaciones nanoestructuradas se ha observado que la dilución es quien presenta un mayor efecto sobre las características estructurales de estos materiales, debido a que la composición química es quien define principalmente el tamaño de cristalita de la matriz [9]. Sin embargo, la información disponible sobre la soldadura de los nuevos sistemas multicomponentes base $\mathrm{Fe}$ es escasa, por lo que resulta relevante conocer cómo diferentes aportes térmicos modifican la composición química y la evolución estructural, junto a otros aspectos del metal depositado. El propósito del siguiente trabajo fue estudiar la influencia del aporte térmico sobre la geometría del cordón, la dilución, la estructura y la microdureza de depósitos de aleaciones base $\mathrm{Fe}$ nanoestructuradas.

\section{Materiales y métodos}

\subsection{Soldaduras}

El consumible empleado fue un alambre tubular de diámetro 1,6 $\mathrm{mm}$, depositado mediante el proceso semiautomático de soldadura con protección gaseosa, usando Ar- $20 \% \mathrm{CO}_{2}$, con un caudal de gas de $18 \mathrm{~L} / \mathrm{min}$ y una longitud libre del alambre de $20 \mathrm{~mm}$ en todos los casos, en forma mecanizada, empleando un dispositivo Miggytrac. En la tabla 1 se muestra la composición química nominal de metal depositado según fabricante.

Tabla 1. Composición química del metal de aporte puro (\% en peso).

\begin{tabular}{|c|c|c|c|c|c|c|}
\hline $\mathbf{C}$ & $\mathbf{M n}$ & $\mathbf{S i}$ & $\mathbf{C r}$ & $\mathbf{N b}$ & $\mathbf{B}$ & $\mathbf{F e}$ \\
\hline$<2$ & $<2$ & $<2$ & $<18$ & $<6$ & $<6$ & Bal. \\
\hline
\end{tabular}

A fin de poder evaluar el efecto del calor aportado sobre las propiedades del cordón se soldaron 8 cupones con 2 niveles de potencia eléctrica y 4 velocidades de soldadura. Los parámetros se eligieron con el propósito de poder comparar situaciones extremas de aporte térmico y otras que tuvieran igual o similar calor aportado pero obtenidas a partir de distintas potencias eléctricas y velocidades de avance. 
Tabla 1. Parámetros de soldadura.

\begin{tabular}{|c|c|c|c|c|c|}
\hline Identificación & $\begin{array}{c}\text { Tensión de arco } \\
(\mathrm{V})\end{array}$ & $\begin{array}{c}\text { Intensidad de } \\
\text { corriente }(\mathrm{A})\end{array}$ & $\begin{array}{c}\text { Potencia eléctrica } \\
(\mathrm{kW})\end{array}$ & $\begin{array}{c}\text { Velocidad de } \\
\text { soldadura }(\mathrm{mm} / \mathrm{s})\end{array}$ & $\begin{array}{c}\text { Calor aportado } \\
(\mathrm{kJ} / \mathrm{mm})\end{array}$ \\
\hline $\mathbf{L 3}$ & 25 & 200 & 5,0 & 3 & 1,7 \\
\hline $\mathbf{L 5}$ & 25 & 200 & 5,0 & 5 & 1,0 \\
\hline L7 & 25 & 200 & 5,0 & 7 & 0,7 \\
\hline L10 & 25 & 200 & 5,0 & 10 & 0,5 \\
\hline H3 & 35 & 300 & 10,5 & 3 & 3,5 \\
\hline H5 & 35 & 300 & 10,5 & 5 & 1,5 \\
\hline H7 & 35 & 300 & 10,5 & 7 & 1,0 \\
\hline H10 & 35 & 300 & 10,5 & 10 & \\
\hline
\end{tabular}

L: baja potencia elétrica; H: alta potencia elétrica;

Se depositó un cordón de soldadura en posición plana, sobre 8 chapas base de $150 \times 75 \times 12,5 \mathrm{~mm}$ de acero al carbono tipo SAE 1010, como se muestra esquemáticamente en la figura 1 . Los parámetros de soldadura empleados se pueden ver en la tabla 1, así como la identificación empleada y el calor aportado calculado en cada caso. Las distintas probetas se agruparon en dos series denominadas con las letras L (Low) y H (High), asociadas a la potencia eléctrica empleada; el número agregado a la letra corresponde a la velocidad de soldadura utilizada.

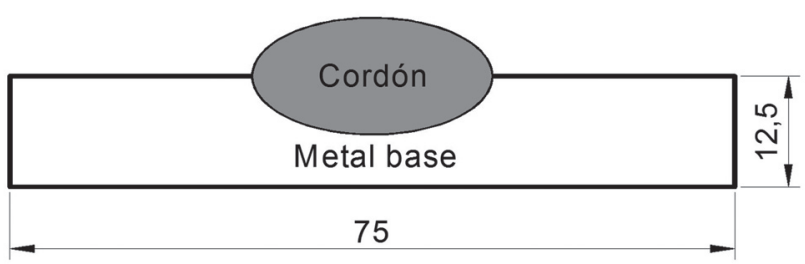

Figura 1. Esquema del cordón; unidades en mm.

De acuerdo con los parámetros de soldadura elegidos, dos pares de probetas fueron soldados con distintas potencia y velocidad de soldadura, pero con similar o igual calor aportado. Son las probetas $\mathrm{H} 7$ y L3 con 1,5 y $1,7 \mathrm{~kJ} / \mathrm{mm}$ de calor aportado, respectivamente y, por otro lado, H10 y L5 ambas con 1,0 kJ/ $\mathrm{mm}$.

Luego de soldadas las distintas probetas se realizó una inspección visual analizándose el aspecto superficial y la

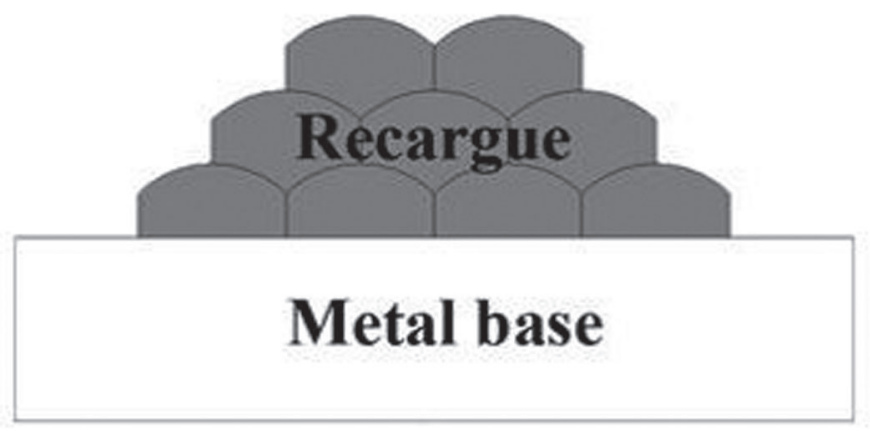

Figura 2. Esquema del cupón de aporte puro. presencia de defectos macroscópicos superficiales.

Por otro lado, a fin de determinar la composición química del depósito libre de dilución, se soldó un cupón de aporte puro para análisis químico (figura 2). La secuencia del mismo fue de 3 capas con 4, 3 y 2 cordones en posición plana. Los parámetros de soldadura utilizados fueron de $300 \mathrm{~A}, 35 \mathrm{~V}$ y $5 \mathrm{~mm} / \mathrm{s}$ de velocidad de avance.

\subsection{Análisis químico y caracterización microestructural}

De cada una de las probetas soldadas se extrajeron cortes transversales que se prepararon para observación metalográfica. Sobre ellos se realizaron mediciones de composición química mediante espectrometría de energía dispersiva de rayos X (EDS). El Boro fue medido mediante la técnica de vía húmeda. Se realizaron mediciones de $\mathrm{Cr}$ y $\mathrm{Nb}$ con EDS sobre cortes transversales a $1 \mathrm{~mm}$ desde la superficie libre del cordón y a $1 \mathrm{~mm}$ de la línea de fusión. Las dimensiones de los cordones: ancho, penetración y sobremonta, se midieron mediante software de análisis de imágenes. Se caracterizó la microestructura mediante microscopía electrónica de barrido (SEM). Sobre los cortes transversales se midió la microdureza Vickers (HV1) a $1 \mathrm{~mm}$ de la superficie, en cinco zonas, promediándose los valores obtenidos. También se tomó microdureza sobre las fases con $\mathrm{HV}_{0,05}$.

Sobre la superficie de cada cordón se realizó difracción de rayos X (DRX), en la zona rayada de la figura 3. El equipo empleado fue un RIGAKU, con radiación de $\mathrm{Cu} \mathrm{K}-\alpha$, entre $35^{\circ}$ y $95^{\circ}$, con una velocidad de barrido de $1 \%$ min. A partir de los espectros obtenidos se analizaron las fases presentes y se determinó el tamaño de cristalita de la fase $\alpha$-ferrita, utilizando la fórmula de Scherrer [10]:

$\beta=\frac{0.9 \lambda}{D \cos \theta}$

Donde $\beta$ es el ancho medio de pico [radianes], $\lambda$ es la longitud de onda $[\AA ̊], \theta$ es el ángulo de Bragg [radianes] y $D$ es el tamaño de cristalita [nm]. 


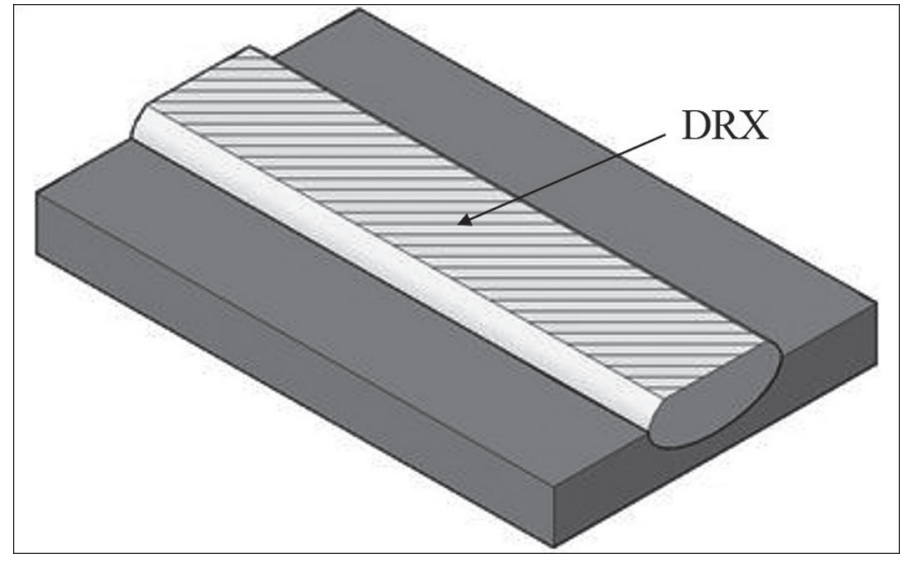

Figura 3. Esquema de la zona de medición de DRX.

La metodología empleada para la cuantificación de las fases por XRD fue mediante bases de datos y factores RIR (Reference Intensity Ratios) [11]. El software utilizado permite hacer estudios cuantitativos precisos por métodos de mínimos cuadrados no lineales sobre el perfil completo del diagrama. La ponderación se consigue con el método RIR normalizado.

\section{Resultados y discusión}

\subsection{Inspección visual}

En la figura 4 se pueden ver las fotos del aspecto superficial de los cordones depositados mediante las distintas configuraciones operativas estudiadas.

Se observó que en todas las probetas soldadas los niveles de salpicaduras y de generación de escoria fueron bajos. Además, los cordones presentaron una buena terminación superficial. En la mayoría de ellos se encontraron fisuras que se generaron durante el enfriamiento del cordón soldado producidas por el alivio de tensiones, típico en estos materiales.

El ancho de los cordones aumentó con el aporte térmico. Los dos juegos de probetas, H7-L3 y H10-L5 soldadas con similar o igual calor aportado y diferentes parámetros operativos, presentaron similar aspecto superficial.

\subsection{Macrografía y análisis dimensional de los cordones}

En la figura 5 se muestran las macrografías de los diferentes cortes transversales de los cupones. Se puede ver tanto el metal base como el depósito, observándose la ausencia de defectos macroscópicos, como poros e inclusiones.

En la tabla 2 se presentan los valores de ancho, penetración y sobremonta medidos sobre cada uno de los cordones depositados.

Puede verse que la geometría del cordón se vio fuertemente afectada por los parámetros de proceso empleados. En la figuras 6 se grafica la evolución del ancho, la penetración y la sobremonta del cordón con la velocidad de soldadura, con cada serie L y H por separado, a fin de ver el efecto de la potencia empleada.
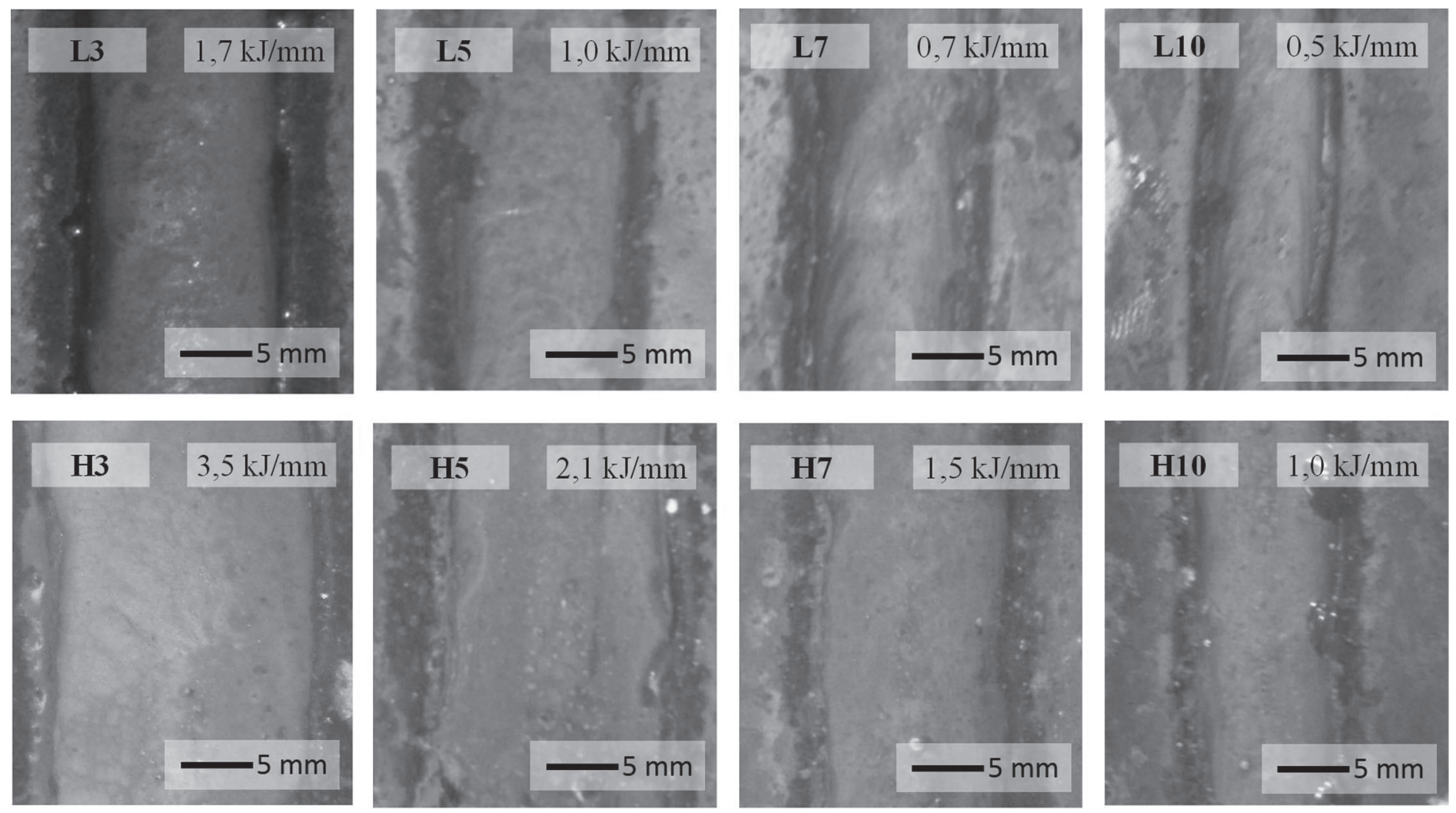

Figura 4. Vista superior de los cordones soldados. 

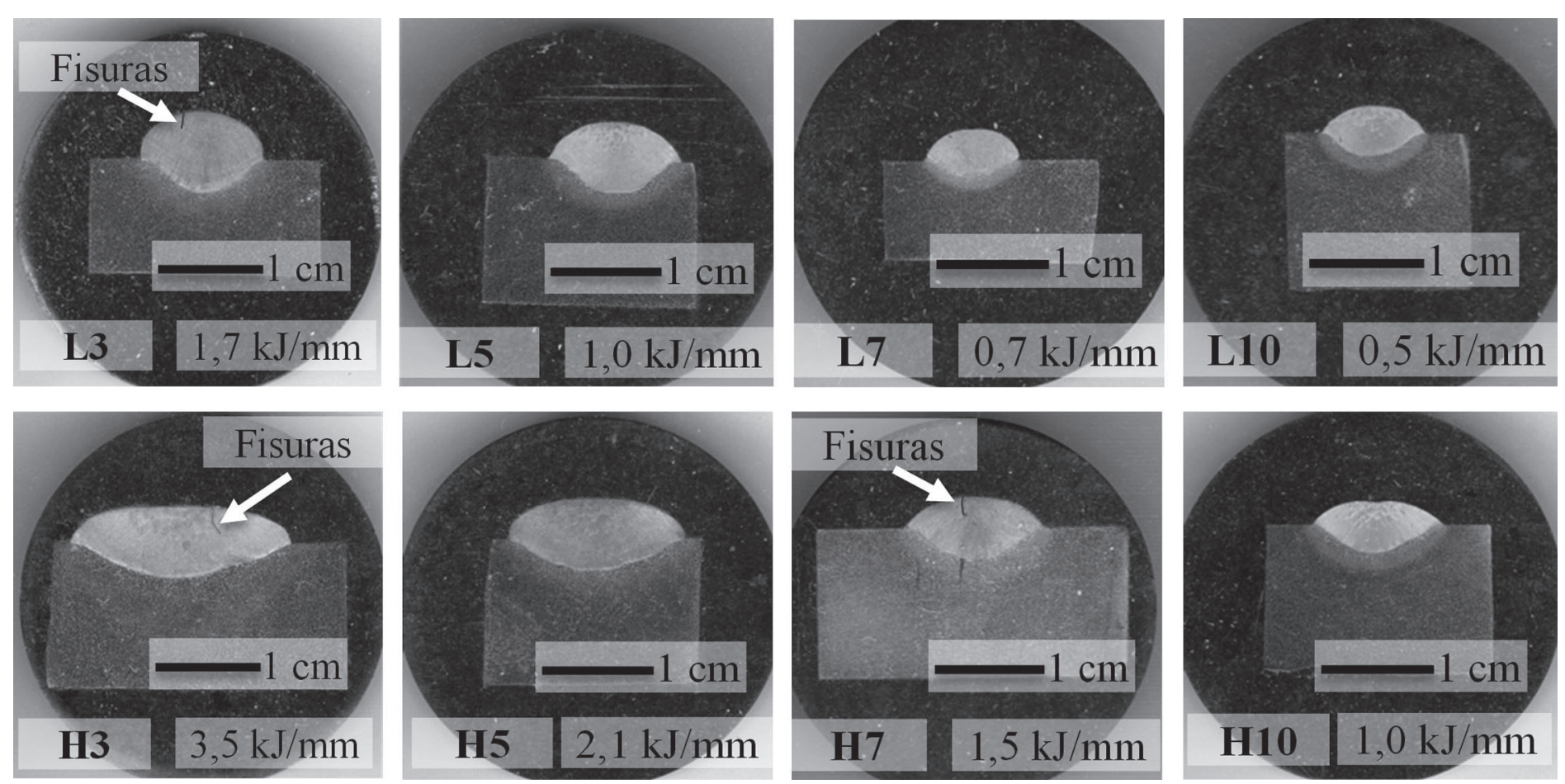

Figura 5. Macroestructura de las probetas soldadas.

Tabla 2. Ancho, penetración y sobremonta medidos sobre los distintos cordones depositados.

\begin{tabular}{|c|c|c|c|c|c|c|c|c|}
\hline Probeta & L3 & L5 & L7 & L10 & H3 & H5 & H7 & H10 \\
\hline Calor aportado $(\mathrm{kJ} / \mathrm{mm})$ & 1,7 & 1,0 & 0,7 & 0,5 & 3,5 & 2,1 & 1,5 & 1,0 \\
\hline Velocidad de avance $(\mathrm{mm} / \mathrm{s})$ & 3 & 5 & 7 & 10 & 3 & 5 & 7 & 10 \\
\hline Ancho de cordón $(\mathrm{mm})$ & 12 & 11 & 9,5 & 8,2 & 18 & 16 & 14 & 11 \\
\hline Penetración $(\mathrm{mm})$ & 3,1 & 2,7 & 2,3 & 2,0 & 3,5 & 3,2 & 2,8 & 2,6 \\
\hline Sobremonta $(\mathrm{mm})$ & 4,0 & 3,9 & 3,7 & 3,2 & 2,8 & 2,6 & 2,5 & 2,0 \\
\hline
\end{tabular}

Tabla 3. Dilución volumétrica para las distintas condiciones de soldadura.

\begin{tabular}{|c|c|c|c|c|c|c|c|c|}
\hline Probeta & L3 & L5 & L7 & L10 & H3 & H5 & H7 & H10 \\
\hline Calor aportado $[\mathrm{kJ} / \mathrm{mm}]$ & 1,7 & 1,0 & 0,7 & 0,5 & 3,5 & 2,1 & 1,5 & 1,0 \\
\hline Dilución (\%) & 35 & 34 & 32 & 30 & 38 & 36 & 36 & 35 \\
\hline
\end{tabular}

El aumento de la velocidad de soldadura produjo una disminución del ancho del cordón y de la penetración, para ambas series. Además, al disminuir la potencia eléctrica, la penetración y el ancho del cordón también disminuyeron, consistentemente con lo esperado. Respecto de la sobremonta se observó que fue mayor para las probetas soldadas con menor potencia eléctrica. Esto estaría asociado a que un aumento de la tensión produjo un aplanamiento del cordón y por ende una disminución en la sobremonta. La velocidad modificó levemente la altura del cordón y esto estaría relacionado con el menor volumen de material aportado.

A su vez, para las condiciones con igual aporte térmico (L3 similar a $\mathrm{H} 7=1,5-1,7 \mathrm{~kJ} / \mathrm{mm}$ y $\mathrm{L} 5=\mathrm{H} 10=1,0 \mathrm{~kJ} / \mathrm{mm}$ ) la geometría del cordón presentó variaciones. Para el caso de 1,5$1,7 \mathrm{~kJ} / \mathrm{mm}$, la probeta $\mathrm{L} 3$ presentó un ancho significativamente menor, con una penetración levemente mayor y una sobremonta fuertemente mayor que la probeta $\mathrm{H} 7$. En cuanto al caso de $1 \mathrm{~kJ} /$ $\mathrm{mm}$, la probeta L5 presentó el doble de sobremonta, con ancho y penetración prácticamente iguales. Estas consideraciones ponen de manifiesto que la geometría del cordón depositado no queda definida por el aporte térmico, sino que las variables que definen dicho aporte deben ser consideradas independientemente. Este aspecto es especialmente relevante en el caso de soldadura de recargue dado que los aspectos geométricos del depósito pueden afectar la dilución y la superficie cubierta, cuestiones que definen la cantidad de capas requeridas y en definitiva aspectos de la calidad y los costos de un dado recubrimiento.

En base a las geometrías de los cordones se calculó la dilución volumétrica de cada muestra [2] mediante software de análisis de imágenes. En la tabla 3 se presentan los resultados.

El porcentaje de dilución varió entre 30 y 38 \% para las distintas 

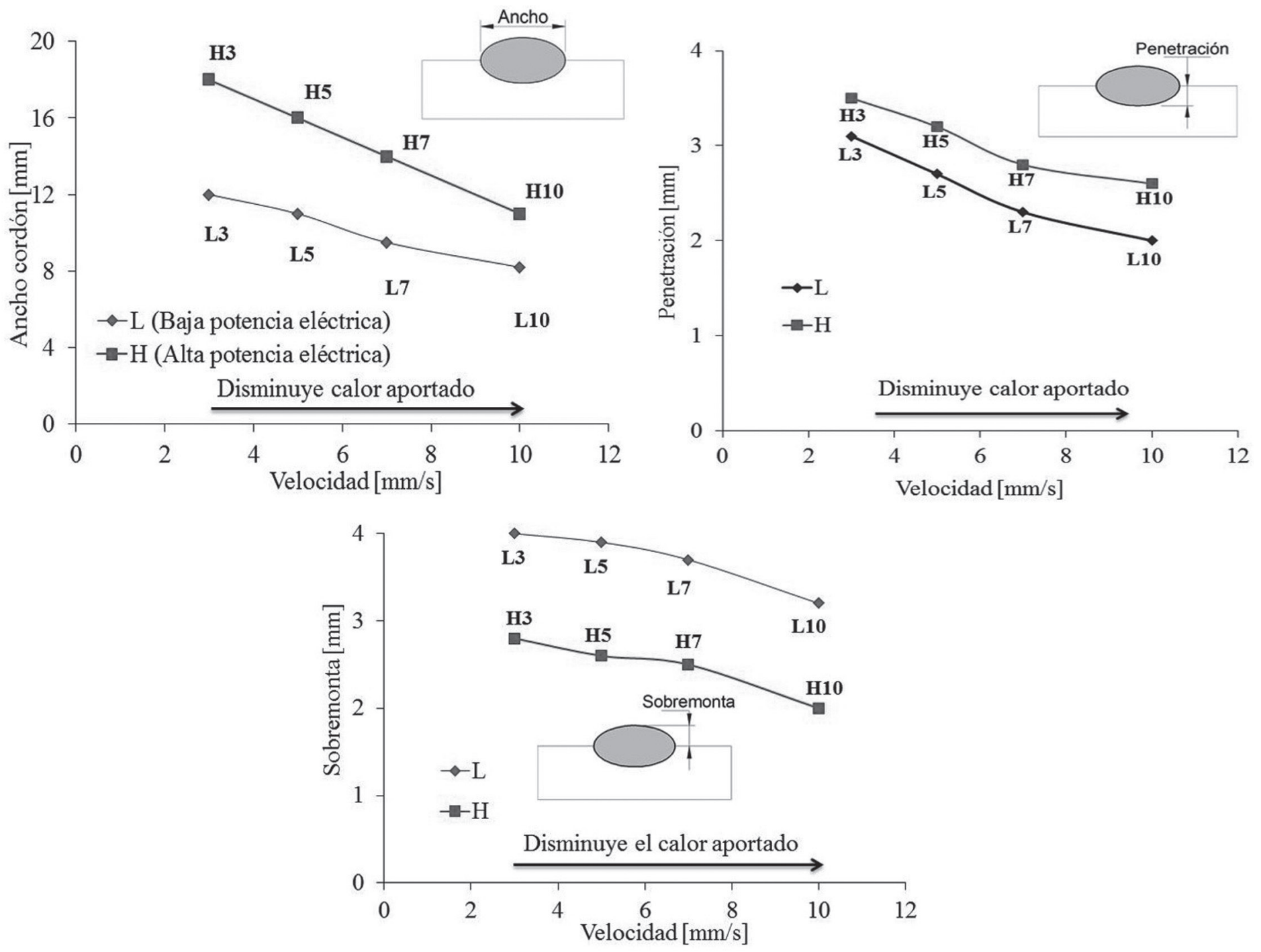

Figura 6. Ancho de cordón y penetración de todas las muestras soldadas.

condiciones de soldadura. Considerando que el aporte térmico fue variado en un factor 7 , el efecto de dicho aporte térmico sobre la dilución puede considerarse bajo. Si bien las variaciones son pequeñas, las muestras soldadas con mayor potencia eléctrica mostraron los mayores valores de dilución. Esto estaría relacionado con la mayor corriente aplicada que produjo mayor penetración. Además, se observó que el incremento de la velocidad de soldadura produjo una disminución de la dilución, lo que de forma similar, estaría asociado a la disminución de la penetración con la velocidad de soldadura. Asimismo, puede verse que para las condiciones con similar aporte térmico (L3/ H7 y L5/H10) no se observó variación de la dilución.

\subsection{Composición química}

En la tabla 4 se muestra el resultado del análisis químico obtenido sobre el cupón de metal de aporte puro.
Tabla 4. Composición química del metal de aporte puro (\% en peso).

\begin{tabular}{|c|c|c|c|c|c|c|}
\hline $\mathbf{C}$ & $\mathbf{M n}$ & $\mathbf{S i}$ & $\mathbf{C r}$ & $\mathbf{N b}$ & $\mathbf{B}$ & $\mathbf{F e}$ \\
\hline 0,99 & 0,22 & 1,02 & 16,8 & 4,6 & 4,6 & Bal. \\
\hline
\end{tabular}

El material depositado presentó una alta concentración de elementos de aleación, dentro del sistema $\mathrm{Fe}-(\mathrm{Nb}, \mathrm{Cr})-$ $(\mathrm{C}, \mathrm{B})$. La composición química cumple con las reglas para la formación de nanoestructuras la cuales son: que el sistema sea multicomponente, con al menos tres elementos de aleación; una diferencia de radio atómico entre los elementos que constituyen el sistema de aleación mayor al $12 \%$ y que el calor de mezcla entre sus elementos tres elementos principales sea negativo [1214]. Estas características producen grandes dificultades para la nucleación y el crecimiento de capas de átomos regulares, generando cristales de tamaño nanométrico.

A fin de evaluar la homogeneidad química dentro del cordón, se realizaron mediciones de $\mathrm{Cr}$ y $\mathrm{Nb}$ con EDS sobre cortes transversales a $1 \mathrm{~mm}$ desde la superficie libre del cordón 
Tabla 5. Concentración de $\mathrm{Cr}$ y Nb (\% en peso) en los distintos cordones depositados.

\begin{tabular}{|c|c|c|c|c|c|c|c|c|}
\hline Probeta & L3 & L5 & L7 & L10 & H3 & H5 & H7 & H10 \\
\hline Calor aportado $[\mathrm{kJ} / \mathrm{mm}]$ & 1,7 & 1,0 & 0,7 & 0,5 & 3,5 & 2,1 & 1,5 & 1,0 \\
\hline $\mathrm{Cr}$ & 10,7 & 10,8 & 11,3 & 11,5 & 10 & 10,4 & 10,6 & 10,9 \\
\hline $\mathrm{Nb}$ & 3,4 & 3,5 & 3,6 & 3,9 & 3,3 & 3,5 & 3,5 & 3,6 \\
\hline
\end{tabular}

y a $1 \mathrm{~mm}$ de la línea de fusión. En estas determinaciones no se encontraron diferencias significativas entre ambos valores $(<5 \%)$, por lo que se puede considerar que la composición química dentro del cordón fue homogénea. Los resultados obtenidos se presentan en la tabla 5.

Se observa que el aumento de la velocidad de soldadura generó un aumento en la concentración de $\mathrm{Cr}$ y $\mathrm{Nb}$ en el depósito. Asimismo, al disminuir el aporte térmico se observó una mayor concentración de $\mathrm{Cr}$ y $\mathrm{Nb}$. Esto estaría relacionado con el nivel de dilución del aporte con el metal base, el cual depende de los parámetros de proceso empleados [2] y con una mayor oxidación de elementos en el arco eléctrico, al aumentar el aporte térmico. En las muestras con similar o igual aporte térmico y distintos parámetros, la concentración de dichos elementos fue similar.

A partir de los datos obtenidos de la composición del metal de aporte puro, del material base y de los cordones depositados se calcularon los valores promedio de dilución de cada probeta. En la figura 7 se muestran los resultados obtenidos junto con los valores de dilución volumétrica determinados en la tabla 2.

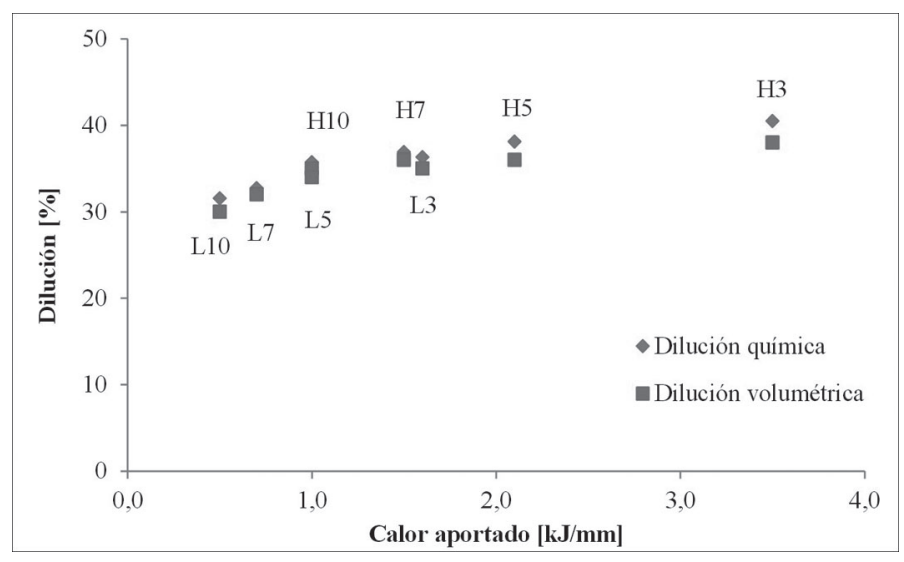

Figura 7. Relación entre las diluciones química y volumétrica y el calor aportado de todos los cordones.

Como se mencionó anteriormente, se observó que al aumentar el calor aportado aumentó la dilución. Esto es consistente con lo observado en los gráficos de penetración y en los cortes transversales.

Los valores de dilución obtenidos por las dos técnicas son muy similares, mostrando la misma tendencia, si bien la dilución volumétrica presentó valores levemente menores. Esto podría estar asociado a que al aumentar el aporte térmico, se tiene un mayor tiempo de solidificación local, por lo que el efecto de oxidación en el arco podría reducir la concentración de los elementos de bajo potencial de oxidación en el depósito [15] generando de este modo mayores valores de dilución química.

En este sentido es interesante la validación hallada de la técnica de medición de la dilución volumétrica respecto de la dilución química, ya que la primera es una técnica sencilla y rápida. Asimismo, el hecho de que la dilución se haya encontrado entre 30 y $40 \%$ para variaciones tan amplias de aporte térmico es también un dato relevante, dada la importancia de la composición química en la formación de materiales nanoestructurados.

\subsection{Caracterización microestructural}

En la figura 8 se muestran los espectros de DRX obtenidos para todas las condiciones. Puede verse que la estructura estuvo formada principalmente por $\alpha-\mathrm{Fe}(56 \%$ (H3) a $49 \%$ (L10)) y carboboruros $\left(\mathrm{M}_{7}(\mathrm{BC})_{3}, \mathrm{M}_{23}(\mathrm{BC})_{6}\right) \quad(38 \%(\mathrm{H} 3)$ a $44 \%(\mathrm{~L} 10))$, detectándose también la presencia de una baja fracción de $\gamma$-Fe y carburo de niobio $(\mathrm{NbC})$ (menor al 1\%) [11].

Asimismo, a partir de estos espectros se determinó el tamaño de cristalita de la matriz $\alpha-\mathrm{Fe}$, que varió de $105 \mathrm{~nm}$ para L10 $(0,5 \mathrm{~kJ} / \mathrm{mm})$ hasta $130 \mathrm{~nm}$ para $\mathrm{H} 3(3,5 \mathrm{~kJ} / \mathrm{mm})$, mínimo y máximo de los calores aportados utilizados, observándose un aumento con el aporte térmico. Estas variaciones podrían estar relacionadas con el porcentaje total de elementos de aleación [14], que disminuye con el aumento del calor aportado y la menor velocidad de enfriamiento asociada a un mayor aporte térmico, lo que podría afectar el crecimiento de los nanocristales.

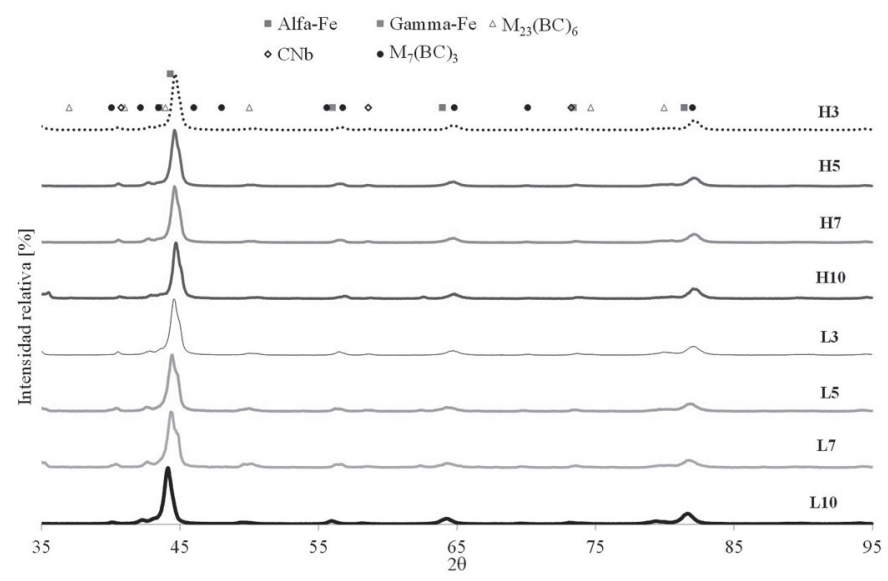

Figura 8. Espectros de XRD de todas las condiciones.

En cuanto a la observación microscópica, en la figura 9 se pueden ver imágenes SEM de las microestructuras obtenidas para distintas condiciones de soldadura.

Se observa un patrón de segregación dendrítico, el cual se vuelve más fino a medida que disminuye el aporte térmico. En la zona interdendrítica se ve una estructura laminar/globular del tipo eutéctica, la cual también se afina al disminuir el aporte térmico. Esta estructura estaría compuesta por $\alpha$-Fe y carboboruros, consistentemente con lo determinado en los espectros de XRD. 

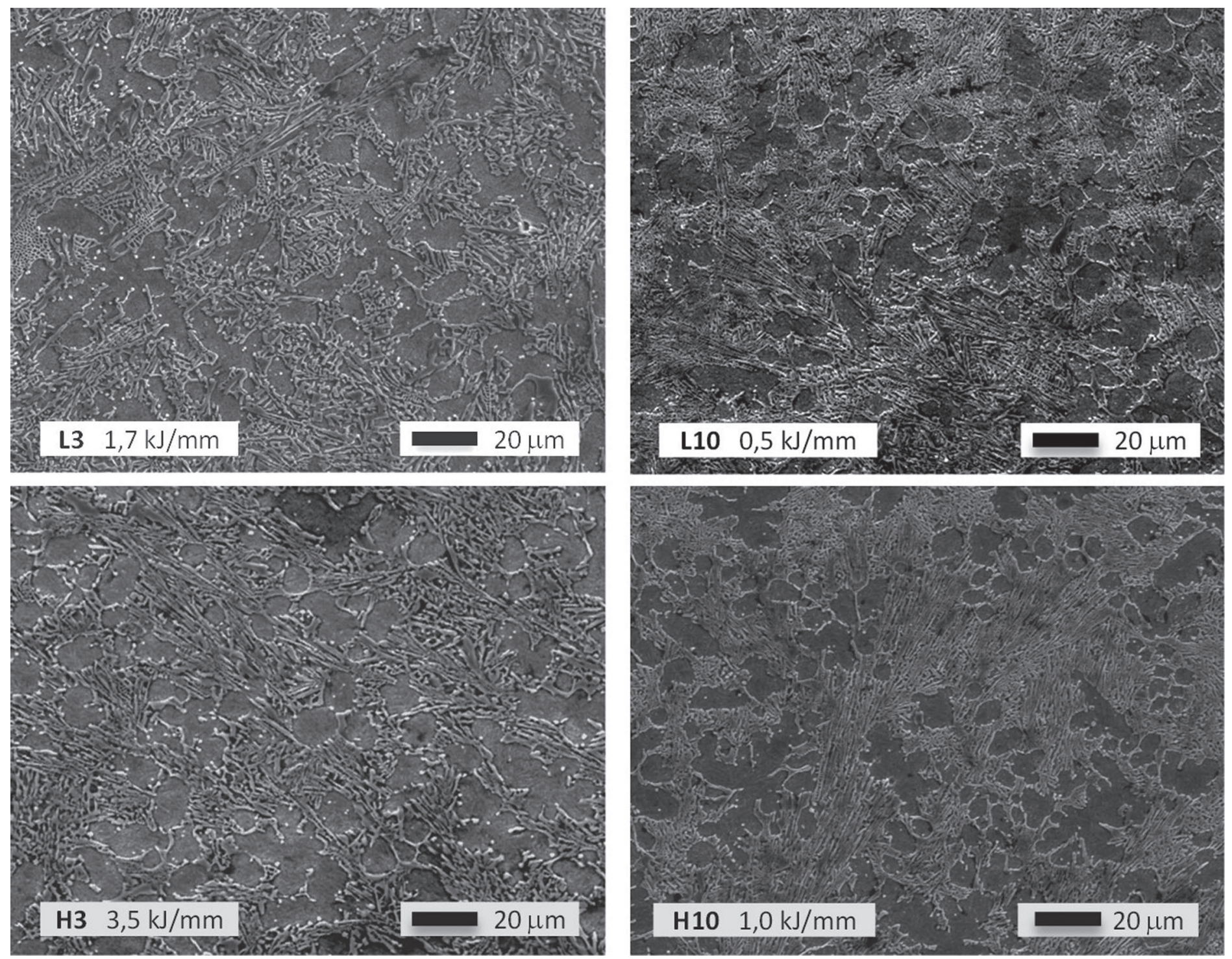

Figura 9. Micrografias SEM de las probetas H3, H10, L3 y L10.

En la figura 10 se muestra un detalle de dicha zona en la probeta H3. La zona interdendrítica indicada como A presenta una mayor concentración de elementos de aleación $(13 \% \mathrm{Cr}, 2 \% \mathrm{Nb})$, mientras que en la zona $\mathrm{B}$ la concentración de $\mathrm{Cr}$ y $\mathrm{Nb}$ disminuyó ( $7 \% \mathrm{Cr}$ y $1 \% \mathrm{Nb}$ ). Esta mayor concentración de aleantes en la zona A es consistente con la ubicación interdendrítica de dicha zona, la cual estaría enriquecida en dichos elementos debido a que correspondería al último líquido en solidificar. Se observó la fracción de eutéctico aumentó al disminuir el calor aportado.

\subsection{Microdureza}

En la tabla 6 se observan los resultados de la microdureza medidos, promedio de por lo menos tres mediciones. Puede verse que los valores variaron entre 806 y $862 \mathrm{HV}$, siendo consistentes con lo esperado para este material $[12,16]$. Para las probetas con aporte térmico similar se obtuvieron durezas equivalentes. Se observó un aumento de la microdureza al disminuir el aporte

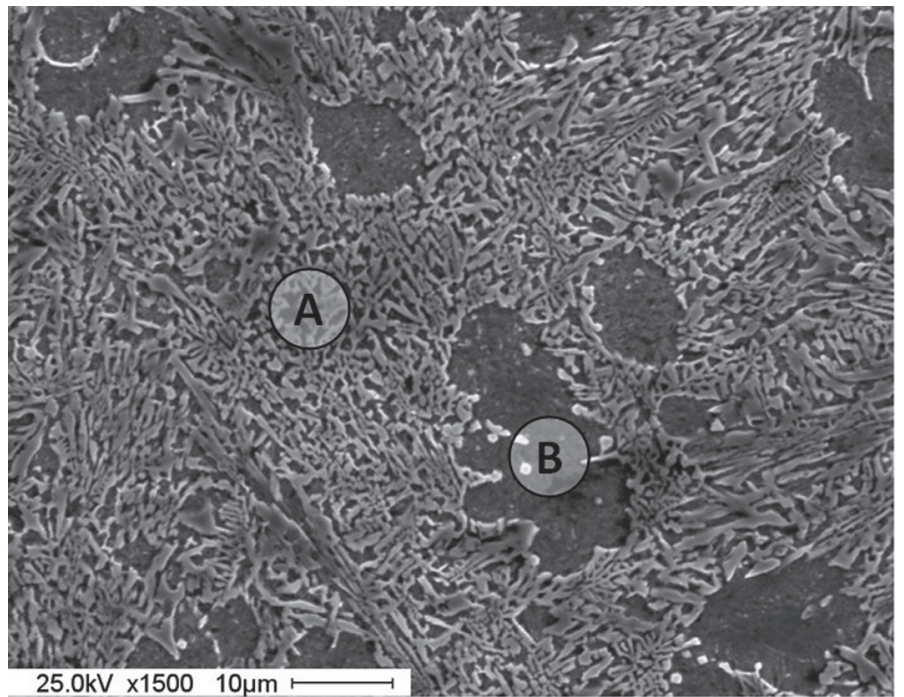

Figura 10. Detalle de la microestructura del cupón H3. 
Tabla 6. Microdureza [HV1kg].

\begin{tabular}{|c|c|c|c|c|c|c|c|c|}
\hline Probeta & L3 & L5 & L7 & L10 & H3 & H5 & H7 & H10 \\
\hline Calor aportado [kJ/mm] & 1,7 & 1,0 & 0,7 & 0,5 & 3,5 & 2,1 & 1,5 & 1,0 \\
\hline Dilución [\%] & 35 & 34 & 32 & 30 & 38 & 36 & 36 & 35 \\
\hline Dureza [HV] & $838 \pm 5$ & $842 \pm 6$ & $850 \pm 5$ & $862 \pm 4$ & $806 \pm 6$ & $836 \pm 7$ & $841 \pm 8$ & $847 \pm 5$ \\
\hline
\end{tabular}

térmico. Este aumento estaría asociado al menor tamaño de cristalita y mayor contenido de elementos en solución producto de la menor dilución. En la figura 11 se grafica la microdureza en función del tamaño de cristalita y del calor aportado.

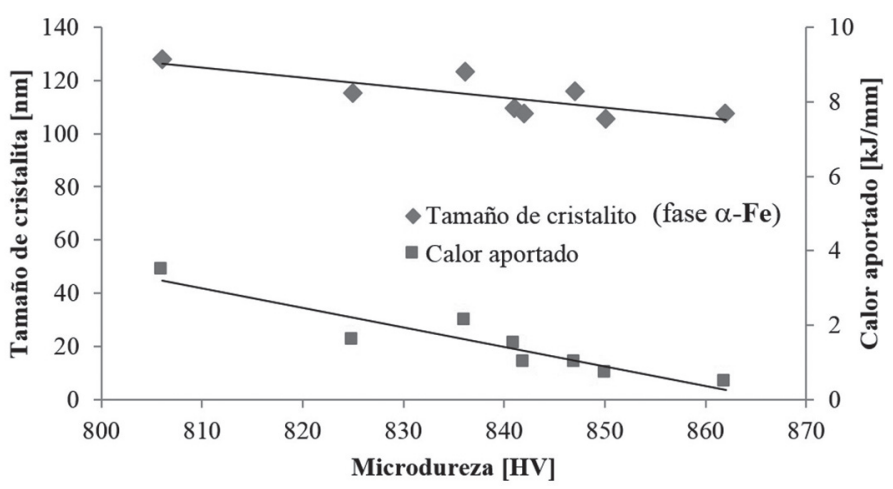

Figura 11. Relación entre tamaño de cristalita, microdureza y calor aportado para las condiciones.

Asimismo, se determinó la microdureza en ambos microconstituyentes observados (matrix de $\alpha$-Fe y estructura eutéctica). Los valores promedio determinados fueron para la matriz $\alpha-\mathrm{Fe}$ de $749 \mathrm{HV}_{0,05}$ y para la estructura globular de $949 \mathrm{HV}_{0,05}$. Dado que la fracción de la fase $\alpha$-Fe, más blanda, aumenta con el aporte térmico, esto podría explicar la disminución de la dureza con el calor aportado. Adicionalmente, como se mencionó anteriormente al disminuir el aporte térmico se produjo una disminución del tamaño de cristalita de la matriz de $\alpha$-Fe y una disminución de la dilución con el consiguiente aumento de los elementos de aleación, lo que podría explicar el aumento de dureza observado. La existencia de una relación entre el tamaño de cristalita y la microdureza ha sido reportada previamente en la literatura para materiales nanoestructurados [16-20].

\section{Conclusiones}

En el presente trabajo se evaluó el efecto de los parámetros de soldadura sobre las características de los depósitos obtenidos de una aleación nanoestructurada base $\mathrm{Fe}$, depositada mediante un alambre tubular bajo protección gaseosa con aportes térmicos entre 0,5 y $3,5 \mathrm{~kJ} / \mathrm{mm}$.

Se concluyó que:

- Todas las probetas soldadas presentaron una buena terminación superficial, bajos niveles de salpicaduras y de escorias. La mayoría de ellas fisuraron durante el enfriamiento.

- El ancho del cordón, la penetración y la sobremonta de los cordones depositados aumentaron con el calor aportado. Para un mismo aporte térmico se observó que la geometría de los cordones fue diferente en función de los parámetros empleados, principalmente la sobremonta, la cual disminuyó con el aumento de la potencia eléctrica.

- La dilución medida a través de la variación de la composición química y del análisis volumétrico, presentó valores similares, validando la técnica de determinación volumétrica, la cual es más sencilla y rápida. El material depositado presentó una dilución de $30 \%$ para el menor aporte térmico $(0,5 \mathrm{~kJ} / \mathrm{mm})$ y de $38 \%$ para el mayor aporte térmico $(3,5 \mathrm{~kJ} / \mathrm{mm})$.

- La microestructura estuvo formada por una matriz de $\alpha$-Fe y carboboruros $\left(\mathrm{M}_{7}(\mathrm{BC})_{3}, \mathrm{M}_{23}(\mathrm{BC})_{6}\right)$, con fracciones menores de $\gamma$-Fe y $\mathrm{CNb}$. Se observó una estructura del tipo eutéctica formada por $\alpha-\mathrm{Fe}$ y carboboruros. La fracción de esta estructura disminuyó con el aumento del aporte térmico.

- El tamaño de cristalita de la matriz de $\alpha$-Fe aumentó con el aporte térmico desde 106 a $130 \mathrm{~nm}$. Este aumento del tamaño de cristalita se puede deber a la disminución del contenido de aleación del depósito al aumentar el calor aportado.

- La dureza aumentó con la disminución del aporte térmico desde 806 hasta $862 \mathrm{HV}$, para los valores extremos de calor aportado analizados. Este aumento de la microdureza estaría asociado al endurecimiento por solución sólida y al menor tamaño de cristalita de la matriz $\alpha-\mathrm{Fe}$, y al aumento de la cantidad de estructura eutéctica de mayor duereza.

\section{Agradecimientos}

Los autores agradecen a EUTECTIC-CONARCO Argentina por la provisión del consumible utilizado, a AIR LIQUIDE Argentina por la donación de los gases de soldadura, a EUTECTIC-USA por la realización de los análisis químicos, al LABORATORIO DE MICROSCOPÍA ELECTRÓNICA DE INTI - MECÁNICA por la realización de la microscopía electrónica de barrido y a APUENFI (Asociación de Profesores Empresarios Área Mecánica de la Facultad de IngenieríaUniversidad Nacional de Lomas de Zamora) por el apoyo económico al presente proyecto.

\section{Referencias bibliográficas}

[1] LINNERT, G.E., Welding metallurgy carbon and alloy steels. 4 ed. AWS: Miami, Florida. 1994. 474 p.

[2] MERRICK, S.; KOTECKI, D.; WU, J., Materials and applications - Part 2. Welding Handbook, American Welding Society. 1998.

[3] HEATH, G., Nanotechnology and Welding - Actual and possible future applications, Proceedings of the CASTOLINEUTECTIC SEMINAR, Brussels: Belgium, 2006. p.25-25. 
[4] ALLEN, S; EDWIN, T., The Structure of Materials. New York: John Wiley \& Sons, Inc. 1999.

[5] KLIMPEL, A; JANICKI, D., A study of worn wear plates of fan blades of steel mill fumes suction system, Proceedings of the 13th Scientific International Conference, Achievements in Mechanical and Materials Engineering" AMME'2005, Polonia: Gliwice, 2005. p.307-310.

[6] EVANS, G.M., The effect of heat input on the microstructure and properties of $\mathrm{C}-\mathrm{Mn}$ all-weld-metal deposits. Welding Journal, v.61, n.4, p.125s-132s. 1982.

[7] GUALCO, A.; SVOBODA, H.G.; SURIAN, E.S.; De VEDIA, L. Effect of welding procedure on wear behaviour of a modified martensitic tool steel hardfacing deposits. Materials \& Design, v.31, n.9, p.4165-4173. 2010.

[8] FRANCIS, J.A.; Principles for open-arc weld deposition of high-chromium white iron surface layers. Adelaide Editor. 1999. [9] WEISSMIILLER J. Alloy effects in nanostructures. Nanostructured Materials, v. 3, p.261-272. 1993.

[10] CULLITY, B.D.; STOCK, S.R. Elements of x-ray diffraction. 3 ed. Prentice Hall. 2001. 520 p.

[11] Software de análisis de espectros de difracción Xpowder, versión 2004.

[12] Datasheet: TeroMatec 395NOA, Eutectic Castolin-ESAB, USA, 2008.

[13] GLEITER, H., Nanostructured materials: basic concepts and microstructure. Acta Materialia, v.48, n.1, p.1-29. 2000.

[14] INOE, A. Amorphous and nanocrystalline materials: Preparation, properties, and applications. Springer, p.206, 2010. [15] VAIDYA, V. Shielding gas mixtures for semiautomatic welds. Welding Journal, v.81, n.9, p.43-48. 2002.

[16] FOUGERE G.E., WEERTMAN J.R.; SIEGEL R.W. On the hardening and softening of nanocrystalline materials. Nanostructured Materials Journal, v.3, n.1-6, p. 379-384. 1993.

[17] WEISSMIILLER J. Some basic notions on nanostructured solids. Materials Science and Engineering, A v.179-180, p.102107. 1994.

[18] MORRIS D.G. The origins of strengthening in nanostructured metals and alloys. Revista de metalurgia, v.46, n.2, p.173-186. 2010.

[19] INOE, A. Bulk glassy and nonequilibrium crystalline alloys by stabilization of supercooled liquid: fabrication, functional properties and applications (Part1). Proc. Japan Acad., v.81, Ser. B. 2005.

[20] BRANAGAN, D.J.; MARSHALL, M.C.; Meacham, B.E., High toughness high hardness iron based PTAW weld materials. Materials Science and Engineering A 428, p. 116-123, 2006. 